\title{
Fundamental Considerations: Impact of Sensor Characteristics, Application Environments in Wireless Sensor Networks
}

\author{
Dongmin $\mathrm{Choi}^{\dagger}$, llyong Chung ${ }^{++}$
}

\begin{abstract}
Observed from the recent performance evaluation of clustering schemes in wireless sensor networks, we found that most of them did not consider various sensor characteristics and its application environment. Without considering these, the performance evaluation results are difficult to be trusted because these networks are application-specific. In this paper, for the fair evaluation, we measured several clustering scheme's performance variations in accordance with sensor data pattern, number of sensors per node, density of points of interest (data density) and sensor coverage. According to the experiment result, we can conclude that clustering methods are easily influenced by POI variation. Network lifetime and data accuracy are also slightly influenced by sensor coverage and number of sensors. Therefore, in the case of the clustering scheme that did not consider various conditions, fair evaluation cannot be expected.
\end{abstract}

Key words: Performance Evaluation of Sensor Network, Sensor Characteristics, Application Environment of Sensor Network

\section{INTRODUCTION}

In wireless sensor networks, sensor nodes are limited disposable battery driven devices [1]. Because of density of sensor data and location $\mathrm{du}^{-}$ plication of sensor nodes in sensor field, sensor data easily and frequently can be duplicated. Thus, in order to prevent unnecessary energy consumption, data redundancy avoidance technology

※ Corresponding Author: Ilyong Chung, Address : (501759) Chosun Univ., Seoseok-dong, Dong-gu, Gwangju, Korea, TEL : +82--62-230-7712, FAX : +82-62-2307754 ,E-mail: iyc@chosun.ac.kr

Receipt date: Dec. 31, 2013, Revision date: Feb. 6, 2014 Approval date: Mar. 19, 2014

${ }^{+}$Division of Undeclared Majors, Chosun University jdmcc@chosun.ac.kr

${ }^{++}$Department of Computer Engineering, Chosun University ※ This study was supported (in part) by research funds from Chosun University, 2013.

※ An earlier version of this paper [20] was published as "Impact of Sensor Data Patterns on Performance Evaluation of Clustering Schemes," Sensor Letters, Vol. 11, 249 258, 2013. is required. In addition, most part to the energy consumption of the sensor node is caused by sensing, data processing, and communication activities $[2,3]$. According to the evaluation result, communication cost was known to be higher than the others $[4,5]$. Therefore, most of the research papers are focused on communication cost reduction method to prolong the sensor network lifetime [6-8]. Generally, routing methods based on network structure are divided into two main groups named flat and hierarchical. Unlike flat based structure, hierarchical structure, using clustering scheme, has proven to have considerable savings in total energy consumption even though the energy consumption is concentrated on the cluster head node. According to the previous research, energy concentration problem is can be relieved through the periodic setup process. Consequently, clustering scheme showed a much better lifetime in performance evaluation. Recently, several cluster based methods uses sensor coverage in terms of cluster 
formation and data transmission. In such a case, if the sensor coverage is wide, there is an advantage where the number of sensor node required on the network is decreased. Therefore, the network lifetime is prolonged. In addition, some kind of methods uses the data comparison scheme, which can decide the data transmission activities in terms of cluster data transmission. In other words, every sensor node on the network can control the data flow through the data comparison scheme which can compare the sensor data value at the current time of the data value at the previous time. Therefore, in the case of appropriate application environment such as the air-temperature collection of the arctic region, the network lifetime is $\mathrm{ex}^{-}$ tremely prolonged. In this way, clustering method in wireless sensor networks showed that it was developed gradually. Nevertheless, most of the clustering methods did not consider about sensor network has particular characteristics in their performance evaluation. One of them is sensor characteristic, and the other one is application environment. Therefore, the experimental results without considering particular characteristics, which are the elements affecting the sensor network operation are difficult to trust. In this work, by using several former clustering methods, we measured the network performance variation that was performed considering sensor characteristics and application environment. According to the performance evaluation result, sensor characteristics seem to influence network performance.

The remainder of this paper is organized as follows: In section 2, we describe related works, also explain our measurements in section 3 . In section 4, we compare data collection rate, network connection rate, energy balance, node isolation rate and network lifetime. Finally, we conclude the paper in Section 5.

\section{RELATED WORKS}

As shown in figure 1, for development of fair evaluation methods, first we classified the cluster-

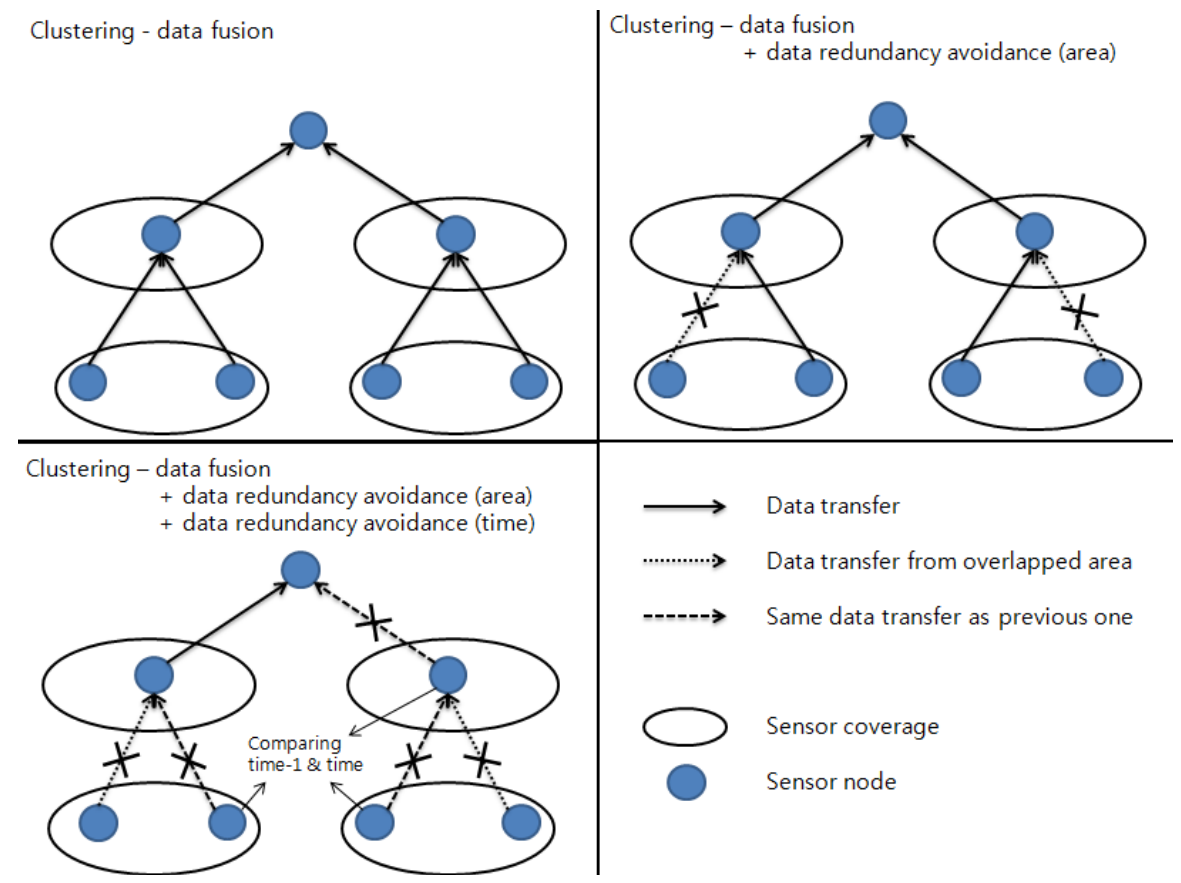

Fig. 1. Clustering method classification. 


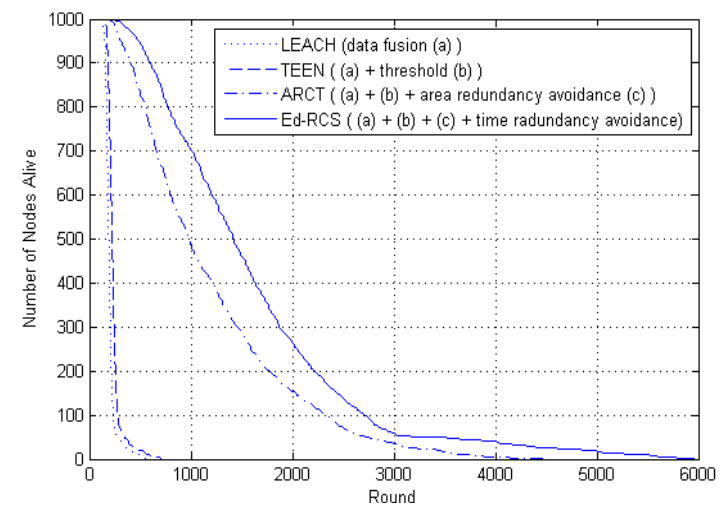

Fig. 2. Network lifetime comparison in accordance with the clustering method classification

ing methods in accordance with the data collection scheme. Clustering methods are categorized into three domains: data fusion, data fusion with area data redundancy, data fusion with area \& time data redundancy. In data fusion clustering, actually that means hierarchical structure; sensor nodes send their data to the sink via intermediate nodes support. During the transmission of sensor data, intermediate nodes can control their burden to conserve energy and to relieve network congestion. Thus, the various schemes to control sensor data were proposed for energy-efficient network operation. As shown in figure 2, each domain shows their network lifetime graph as a showcase of energy conservation.

\subsection{Data fusion clustering}

LEACH (low-energy adaptive clustering hierarchy) [9] is a representative clustering method in sensor networks. In clustering process, cluster head node's aggregate data from their in-cluster nodes. Then combine, retransmit to the Base station or sink in periodic time. According to the evaluation assumption, did not consider sensor characteristics and application environment. Therefore, we assumed that this scheme can be applied in the various environments. In $\mathrm{LEACH}^{-} \mathrm{C}$ (low-energy adaptive cluster hierarchy centralized) [9], to solve the problem of repositioning cluster head nodes, clusters are elected by a base station at every round. In this method, every sensor node sends the message which includes node's position and residual energy information to the base station. After that, base station broadcasts this information to all the nodes, which are deployed in the field help to form an adequate cluster. This scheme has no mention about sensor characteristics and application environment. Therefore, we assumed that this scheme can be applied in the various environments. HEED (hybrid, energy-efficient distributed) [10] requires some values of node's residual energy for cluster formation and election. In this scheme, more residual node energy means more probabilities of being elected as a cluster header node. If candidate nodes have same residual energy, then their transmission costs are compared. In the case of assigning the complex calculations to the base station, BCDCP (base station controlled dynamic clustering protocol) [11] is same as $\mathrm{LEACH}^{-} \mathrm{C}$. This scheme is composed of two phases: setup and data communication. In cluster formation, base station elects candidate set of cluster head nodes to $\mathrm{de}^{-}$ termine cluster head nodes. To fit the number of cluster head nodes, it uses a cluster splitting algorithm which divides the network continuously. HEED and BCDCP did not mention about sensor characteristics and application environment. Therefore, we assumed that these are can be applied in the various environments. Based on LEACH, in TEEN (threshold sensitive energy-efficient sensor network protocol) [12] sensor nodes manage threshold value. After cluster formation, cluster head nodes transmit the data parameters, HT (hard threshold value), and ST (soft threshold value), to their member nodes. Then, every node collects and transmits data when the value exceeds the HT value first. After exceeding HT, data collection and transmission perform only when the measured data exceeds ST. APTEEN (A Hybrid Protocol for Efficient Routing and Comprehensive Information Retrieval in Wireless Sensor Net- 
works) [13] is a hybrid protocol that unites the data transmission by the threshold value of TEEN and the periodic data transmission of LEACH. Even the collected data did not exceed threshold values; by periodic data transmission schedule, every node transmits collected data to the cluster head node. According to their performance evaluation, TEEN and APTEEN assumed that simple temperature sensing application. Therefore, we assume that TEEN and APTEEN are application specific clustering schemes.

\subsection{Area redundancy avoidance clustering}

ARCT (An Advanced Regional Clustering Scheme using Threshold dataset) [14] is a protocol based on the dynamic cluster that performs upon the basis of the cluster and proactive network that transmits at the determined time. In addition, it uses threshold-similar as TEEN. Therefore, it can be categorized as a reactive network such as TEEN. This scheme uses two types of clusters - the regional cluster and the normal cluster-. These two clusters have different ways to collect data. The regional cluster composes the cluster through the data comparison process which collected by adjacent nodes, and after that only the head node of the regional cluster acts and transmits the collected data. The normal cluster combines with the nodes that failed to participate in the regional cluster. All the nodes that composed this cluster participate in data collection. The nodes that participate in this cluster increase the energy efficiency using the threshold value table when collecting data. This scheme is based on air-temperature data collecting. Therefore, this is an application specific clustering scheme.

ARCS (An Energy Efficient Clustering Scheme for Sensor Network Monitoring Systems) [15] is based on the basic concept of ARCT. In ARCS, every node that forms a cluster needs to consider two types of values. The first is its sensor value, and the second is its altitude. Furthermore, in order to decrease the error rate during data collection, ARCS uses one more condition for a node to be included in a cluster. In order to achieve higher sensor data accuracy, ARCS considers the sensor coverage to form a cluster. According to the performance evaluation, ARCS support environmental monitoring. Therefore, this scheme can be applied in the various environments. EEDCF (Energy Efficient Deployment and Cluster Formation Scheme) [16] is a scheme based on the clusters which divide into predetermined grids. In addition, it uses two different types of nodes to form a cluster. One type of nodes has a higher energy and wider range compared to the other type of nodes. This type is called S1 and the other type is S2. They assumed that $\mathrm{S} 1$ type nodes have twice the energy and radius coverage compared to the S2 type nodes. S1 node which is positioned in the central of the cluster called $\mathrm{CN}$, and this node is not active in the setup phase1. The other S1 nodes are performed as a role of the cluster head node for the first few rounds. After the energy of S1 nodes became almost as much as the energy of the S2 nodes, the S1 nodes stop becoming the $\mathrm{CH}$. After then, the $\mathrm{CN}$ acts as a $\mathrm{CH}$ for the cluster in the setup phase2. All other nodes, including S1 nodes are treated as the S2 type node because they have similar energy to S2 nodes. In this paper, they did not mention about any other application environment. Therefore, we assumed that this scheme can be applied in the various environments.

\subsection{Time \& area redundancy avoidance clustering}

CM-EDR (Continuous-Monitoring Using Event Driven Reporting) [17] is a scheme that each node deals only with its local sensor data and does not aggregate data from member nodes. Each sensor node compares the current sensor data with the last transmitted value to the sink. Data is only transmitted when the value is changed with respect to the data of the sink. This scheme is only 
for the continuous monitoring system. However, there is no restriction in sensor data collection. Therefore, we assumed that this scheme can be applied in the various environments. Ed-RCS (An Energy-aware Event-driven Regional Clustering Scheme) [18] is a scheme that sensor node can predict current data using data prediction graph and to minimize data transmission among the cluster head node and member nodes. Thus, energy-efficient data transmission is possible. According to the assumption, this scheme can be applied in the various environments. Furthermore, in the case of the scheme that is seeking resource conservation through intelligent processing, network performance may be depended on sensor characteristics. Thus, it is recommended that clustering schemes evaluate its performance in various environments for fair evaluation and comparison.

\section{CONSIDERABLE CHARACTERISTICS}

As the considerable items for fair evaluation, we categorize these items into three domains: sensor data density\& sensor coverage, sensor data pattern, multi-sensor data.

\subsection{Sensor data density \& sensor coverage}

The number of data collected in the same area can be expressed as the density. As shown in the

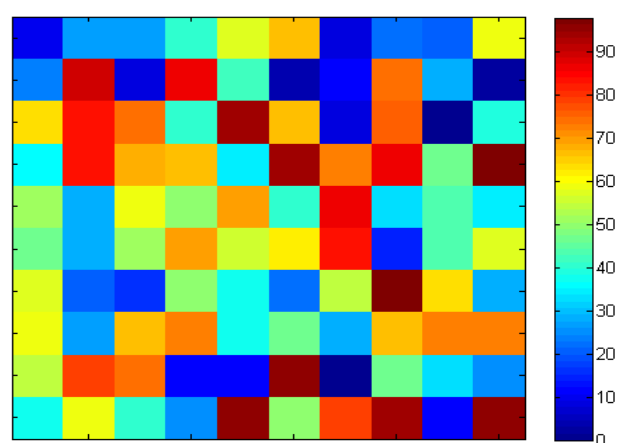

(a) figure 3(a), a few sensor data are detected: that means low density. On the contrary, as shown in the figure 3(b), numerous sensor data is detected: high density. Figure 3 shows comparison graph of detected sensor data in same size of sensor field. For example, 1000 sensor nodes are uniformly deployed in $100 \mathrm{~m}^{2}$ of sensor field. To accomplish $100 \%$ of data detection, in the case of figure 3(a) $10 \mathrm{~m}^{2}$ of sensor data density, it must be guaranteed at least one sensor node deployed in each $10 \mathrm{~m}^{2}$. Therefore, only 100 sensor nodes can support whole sensor field with no errors. However, to accomplish $100 \%$ of data detection, in the case of figure 3(b) $1 \mathrm{~m}^{2}$ of sensor data density, it must be guaranteed at least one sensor node deployed in each $1 \mathrm{~m}^{2}$. Thus, tenfold more sensor nodes are required.

Therefore, we know that the density of sensor data can become the factor affecting on the sensor network operation and organization. In addition, as shown in figure 4 , we assume that same sensor data is detected in the specific area of sensor field. When the maximum sensor detection length $r$ is extended as $2 \mathrm{r}$, sensor node can cover 8 more neighbour sensor nodes. In other words, neighbour nodes do not need to activate their sensor to collect sensor data. Therefore, that means prolonged network lifetime.

According to the sensor data density and cover-

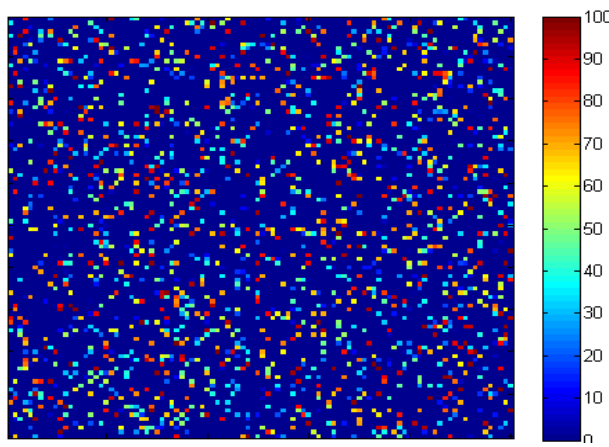

(b)

Fig. 3. Sensor data density comparison in same target area. (a) 100 sensor data spot of $10 \mathrm{~m} 2$ size, and (b) 10000 sensor data spot of $1 \mathrm{~m} 2$ size. 

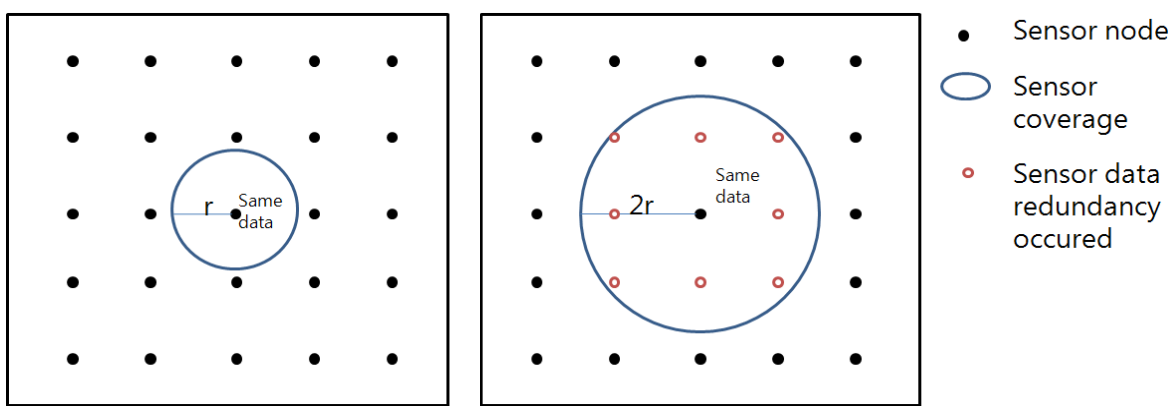

Fig. 4. Sensor coverage comparison.

age, we classified the existing several clustering methods into two categories: sensor coverage and non-sensor coverage consideration. Such as LEACH, TEEN, APTEEN and ARCT, belong to non-sensor coverage consideration, are called classic clustering method. These kinds of methods are based on sensor data processing within transmission range of sensor nodes. Thus, we assumed that sensor nodes of these networks acquire the sensor data from the coordinates of nodes. Therefore, in order to compare the performance of these methods, we measured the network performance in accordance with the sensor data density. Considering the sensor coverage, such as ARCS, Ed-RCS and CM-EDR are called advanced, they form the cluster based on the sensor coverage. Thus, pre-definition of the network application $\mathrm{de}^{-}$ termines the network performance. Therefore, we measured the network performance in accordance with the sensor data density and sensor coverage for each case.

\subsection{Sensor data pattern}

Application specified sensor network have various sensor data patterns according to their specific environment. Therefore, we selected representative five data patterns. These data patterns appeared frequently in the application environment of the previous researches.

\subsubsection{Periodic changing sensor data pattern}

Figure 5 shows periodic changing data pattern,

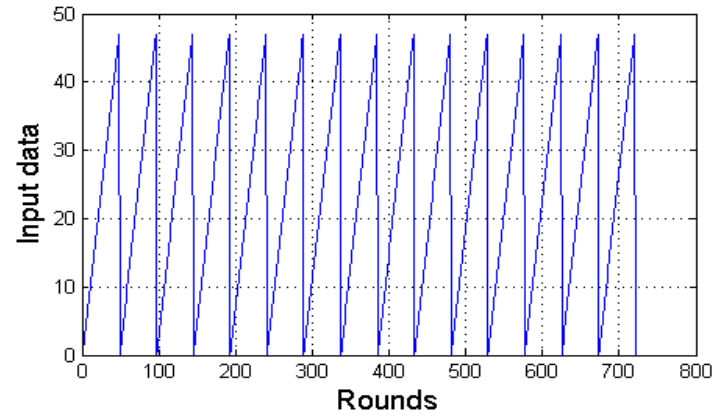

Fig. 5. Periodic changing sensor data pattern.

such as subway circulation or city bus route. In this case, the most important issue is: collecting sensor data with lower energy consumption while maintain high data accuracy.

\subsubsection{Object tracking sensor data pattern}

Sensor network application used for object tracking is completely different from simple environmental monitoring. Many sensor nodes need to keep on collaborating with other nodes in order to track the moving target. In this case, the most important issue is: tracking the target with lowest energy consumption while maintain the low missing-rate. Figure 6 shows moving object and object sensing.

\subsubsection{Air-temperature sensor data pattern}

For the case of simple environmental monitoring, we applied real air-temperature data. In the case of air-temperature monitoring, sensor network needed to maintain the network without data 


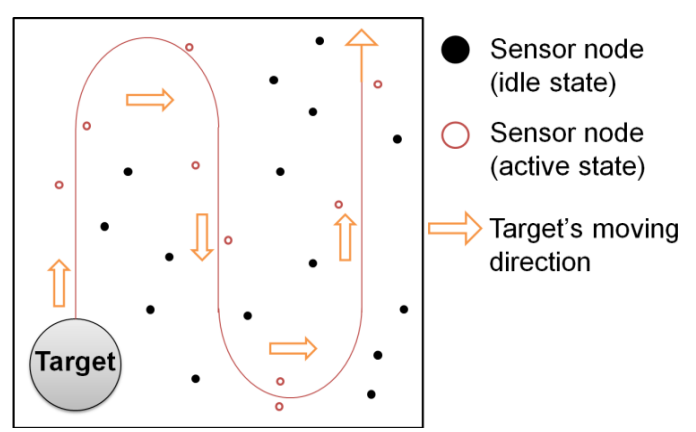

Fig. 6. Target tracking sensor data pattern.

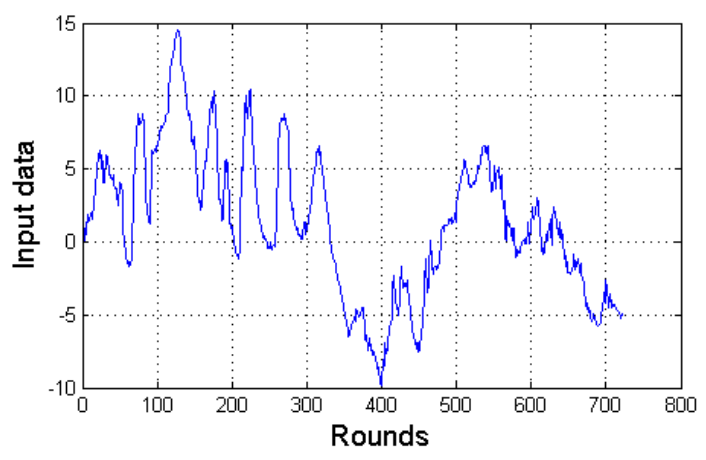

Fig. 7. Air-temperature sensor data pattern.

loss. Figure 7 shows the air-temperature data pattern.

\subsubsection{Random sensor data pattern}

In the worst case, there is no relationship between data and application environment. In this case, most of the routing schemes show an inefficient result. Figure 8 shows randomly generated sensor data pattern.

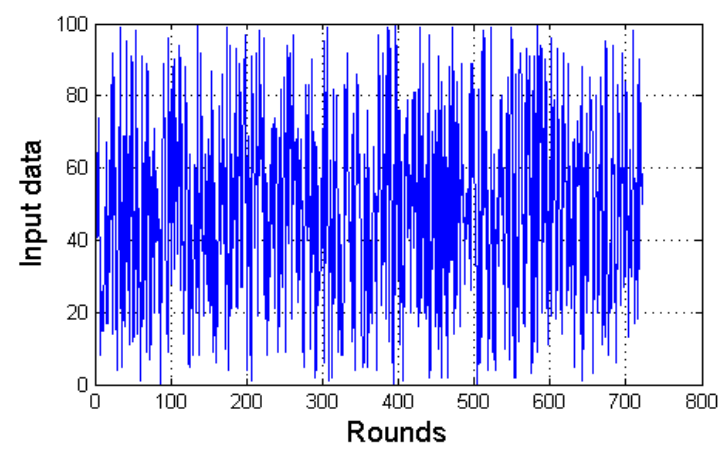

Fig. 8. Random sensor data pattern.

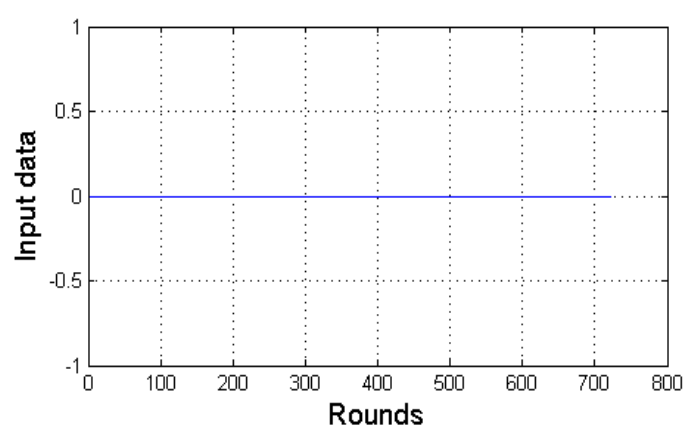

Fig. 9. None sensor data pattern.

\subsubsection{None sensor data pattern}

In the case of disaster monitoring, the network may not sense any data for a long time. In this case, the most important issue is maintaining the network with low energy consumption. Furthermore, sensor alarm message must be transferred without delay while maintain low error rate. Figure 9 shows none data pattern.

\subsection{Multi sensor data}

In normal case, sensor node hardware can support multi-sensor module. However, most of the recent simulation results in research papers did not consider the multi-sensor data pattern. We assume that network performance will be influenced by the multi-sensor. According to the performance evaluation result, number of sensor module on the sensor node seems to have an effect on the network performance.

\section{PERFORMANCE EVALUATION}

In this section, we present the experimental results of clustering scheme in terms of data accuracy, node participation rate for the network, node isolation rate, residual energy and network lifetime in accordance with the random or different sensor data patterns.

\subsection{Network basic assumption}

For the performance evaluation, we considered 
Table 1. Simulation parameters

\begin{tabular}{|ll|l|l|}
\hline \multicolumn{1}{|c|}{ Parameter } & Value & \multicolumn{1}{c|}{ Unit } \\
\hline \hline $\mathrm{E}_{\mathrm{elec}}$ & (electronic energy) & 50 & $\mathrm{~nJ} / \mathrm{bit}$ \\
$\mathrm{E}_{\mathrm{fs}}$ & (amplified energy of freespace model) & 10 & $\mathrm{pJ} / \mathrm{bit}^{2} \mathrm{~m}^{2}$ \\
$\mathrm{E}_{\mathrm{mp}}$ & (amplified energy of multipath model) & 0.0013 & $\mathrm{pJ} / \mathrm{bit} / \mathrm{m}^{4}$ \\
$\mathrm{E}_{\mathrm{schedule}}$ & (consumed energy of node scheduling) & 5 & $\mathrm{~nJ} / \mathrm{bit} / \mathrm{signal}$ \\
$\mathrm{E}_{\mathrm{da}}$ & (consumed energy of data aggregation) & 5 & $\mathrm{~nJ} / \mathrm{bit} / \mathrm{signal}$ \\
$\mathrm{l}$ & (message length) & 1000 & $\mathrm{bit}$ \\
$\mathrm{N}$ & (number of sensor nodes) & 1000 & $\mathrm{ea}$ \\
$\mathrm{T}$ & (node transmission distance) & 50 & $\mathrm{~m}$ \\
$\mathrm{~S}$ & (node sensing distance) & 0 to 50 & $\mathrm{~m}$ \\
$\mathrm{Application}$ environment data & Random & $\mathrm{m}$ \\
$\mathrm{M} \quad$ (length of a side of the network) & 200 & $\mathrm{~m}$ \\
\hline
\end{tabular}

various environmental sensor data described before. Furthermore, we made some assumptions about sensor nodes, networks, and the environment. Basic assumptions are as follows:

Nodes transmit data in a multi-hop fashion. Nodes positions are fixed. Nodes are synchronized according to their time. All nodes have similar capabilities (processing, communication, initial energy). Nodes can adjust their transmission power. Sensor ranges are shorter than their transmission ranges. Base station (or Sink) has no limitations in energy and of transmission coverage. Experiments were conducted using MATLAB simulator. The simulation parameters are summarized in table 1.

\subsection{Data accuracy and lifetime comparison}

With the change of the density (point of interests), we measured the data accuracy in all clustering methods. For example, 100 POI indicates 100 sensor data, which is generated from the sensor field. Figure 10 and 11 show the data accuracy of the clustering method in accordance with the number of POI, sensor coverage and transmission range.

According to the experiment result, we can conclude that classic clustering methods are easily influenced by POI variation.

Moreover, in the case of advanced methods, which are shown in figure 11, network lifetime and data accuracy are slightly influenced by sensor coverage. Capital $\mathrm{C}$ and $\mathrm{T}$ denote sensor coverage and transmission range respectively.

\subsection{Network lifetime comparison}

Figure 12 shows the network lifetime which is classified by each data pattern

According to figure 12 , LEACH is not affected by data patterns. Because LEACH has no algorithm to reorganize sensor data except cluster head node data aggregation. TEEN shows a different result, even though TEEN is based on the LEACH. APTEEN shows a same result compare with TEEN. However, still this network not satisfies user requirement. ARCT shows not a good result about the dead node when it applied random data pattern and air-temperature data pattern. According to the figure, ARCS seems not to be influenced by application environments. ARCS uses sensor coverage to elect active node. From active node, they elect network participants to form clusters. With periodic cluster reformation, these clusters transfer their data during the network lifetime. In CM-EDR, periodic, random, and air-temperature data pattern showed the slightly lower results. In random data pattern, there is no correlation between data. Hence, CM-EDR cannot process this kind of data effectively. Similarly, periodic data pattern and air-temperature data pattern has not been enough correlation in their data. According to the figure, 


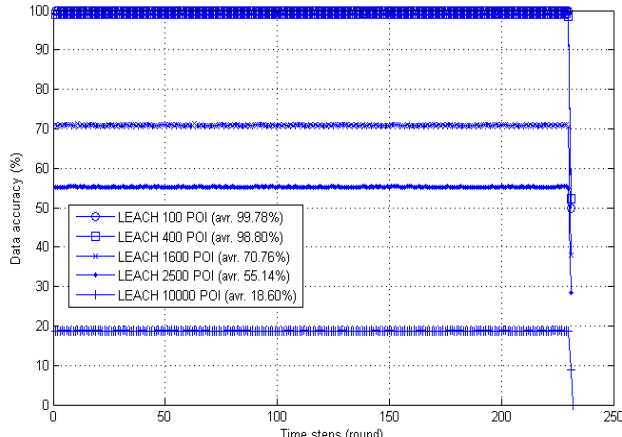

(a)

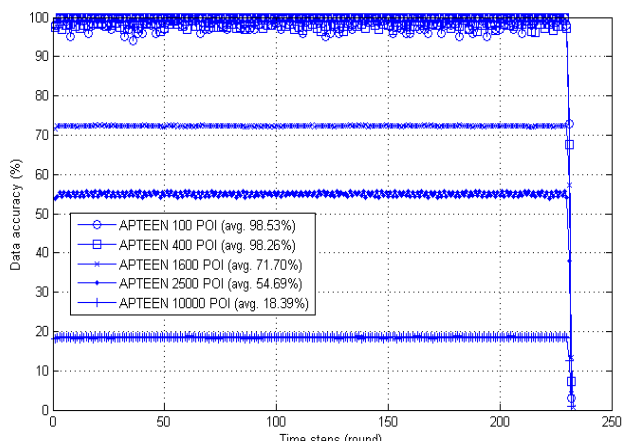

(c)

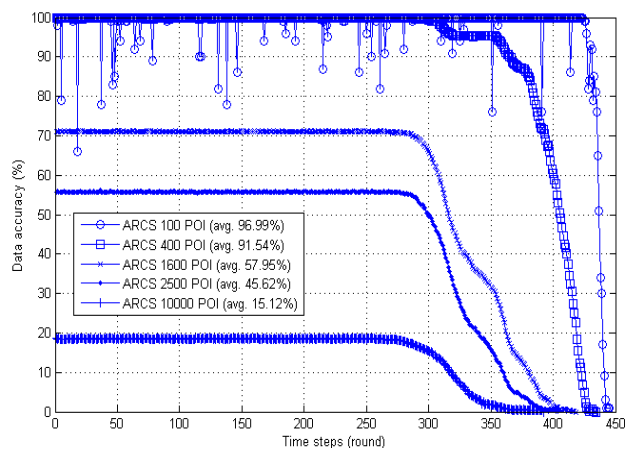

(e)

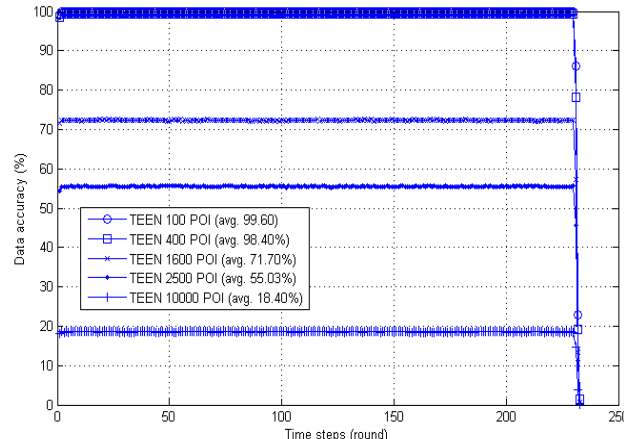

(b)

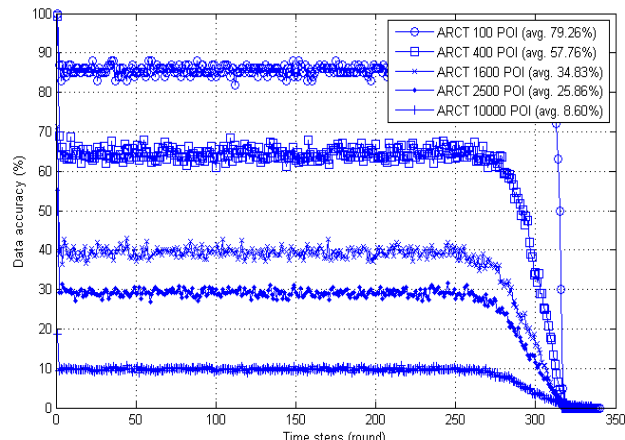

(d)

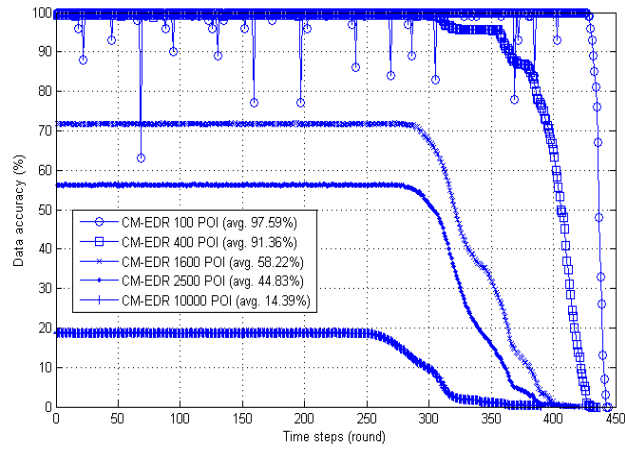

(f)

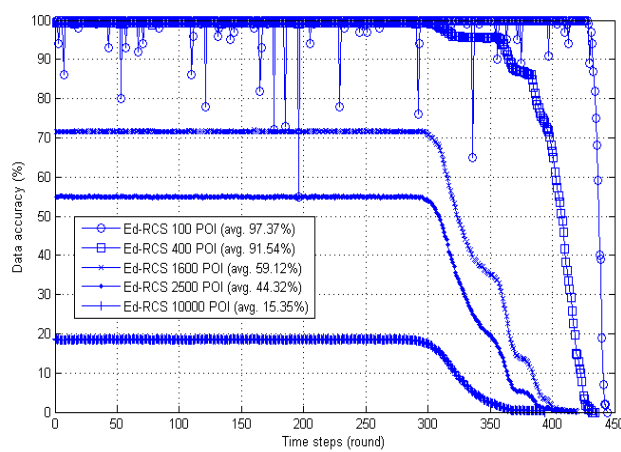

(g)

Fig. 10. Data accuracy and lifetime comparison graph without sensor coverage consideration. (a) LEACH, (b) TEEN, (c) APTEEN, (d) ARCT, (e) ARCS, (f) CM-EDR, and (g) Ed-RCS. 


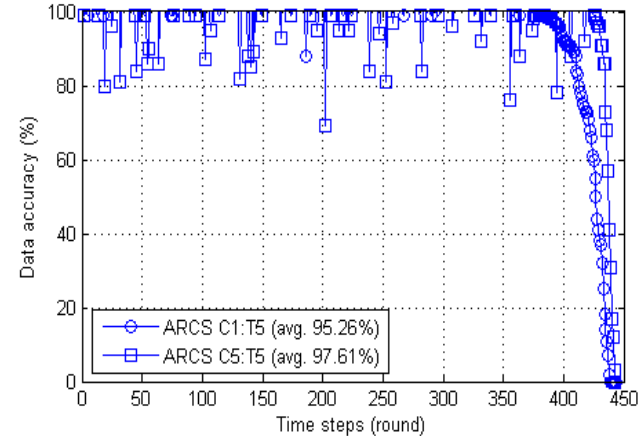

(a)

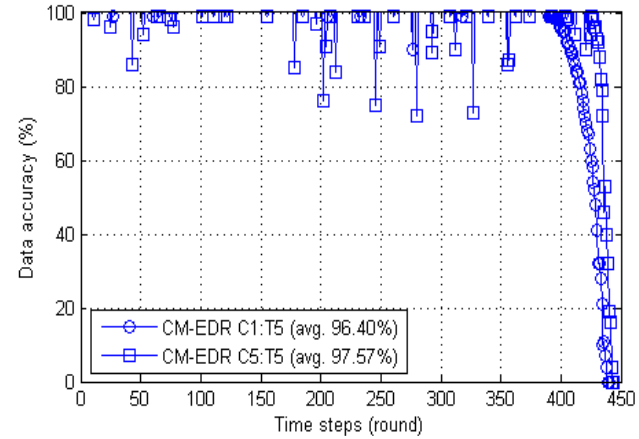

(b)

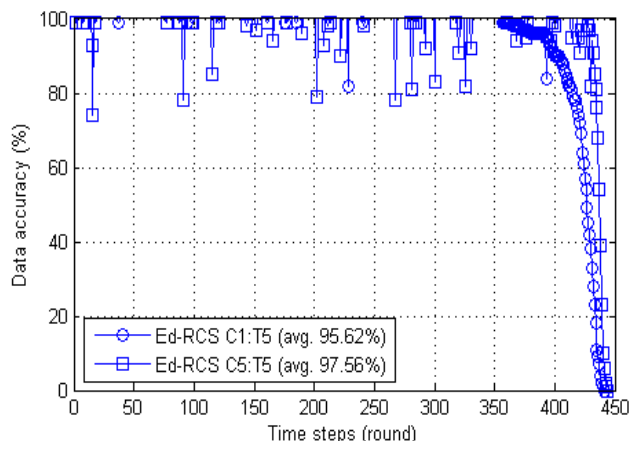

(c)

Fig. 11. Data accuracy and lifetime comparison graph with sensor-coverage consideration. (a) ARCS, (b) CM-EDR, and (c) Ed-RCS.

Ed-RCS shows the better results. Ed-RCS shows the relatively lower results in case of random data pattern and air-temperature data pattern. Basically, this scheme was proposed to achieve high performance in air-temperature application environment.

\subsection{Data accuracy comparison}

Figure 13 shows data accuracy of clustering scheme along with network lifetime.

According to figure 13, LEACH has the highest accuracy. Most of the data show stable data accuracy. Even in the case of air-temperature data pattern, it looks almost stable. Due to the threshold values, TEEN looks unstable. It means that TEEN cannot be trusted and be proven as a good scheme. Same as TEEN, APTEEN shows unstable data $\mathrm{ac}^{-}$ curacy about each data. Especially, in the case of air-temperature data pattern, APTEEN shows se- vere fluctuations in the graph because of periodic transmission. Regardless of data pattern, ARCT shows severe differences in data collection accuracy. ARCS shows no significant difference. CM-EDR and Ed-RCS show similar graph.

\subsection{Node isolation probability comparison}

In a clustering scheme, a cluster head node performs the role of a repeater between the source node and the sink. Thus, the number of cluster head nodes is important. Typically, in cluster header selection using probability, more cluster head nodes mean a higher connection probability.

Figure 14 shows the ratio of the isolation nodes during the network lifetime. Node's isolation is displayed regardless of a kind of data pattern. In the case of ARCT, node isolation is frequently occurred. It is mainly caused by their cluster forming scheme. 


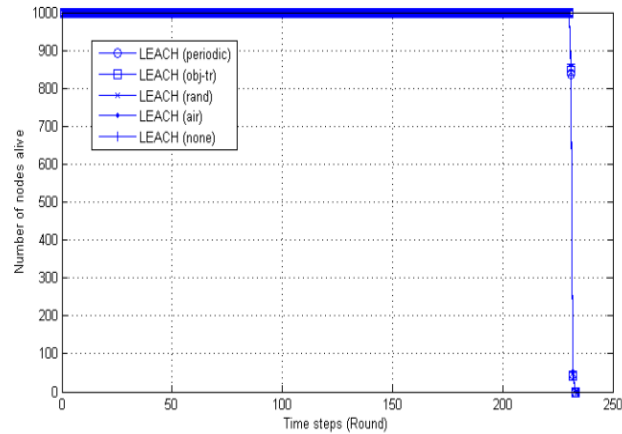

(a)

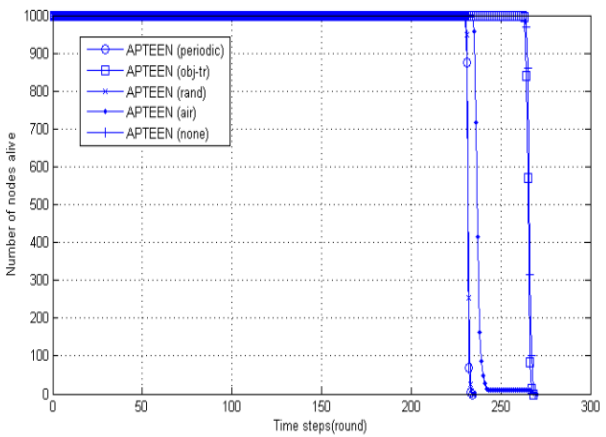

(c)

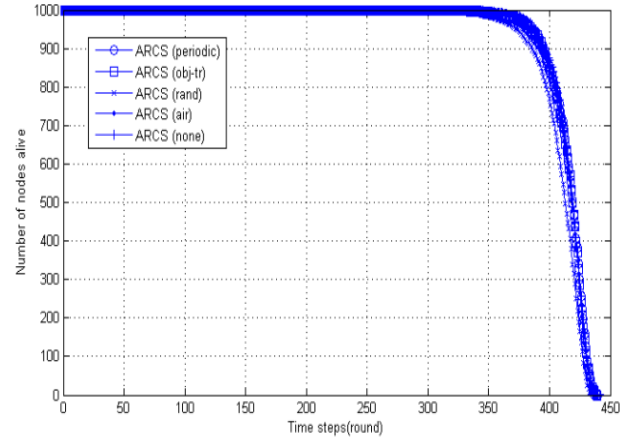

(e)

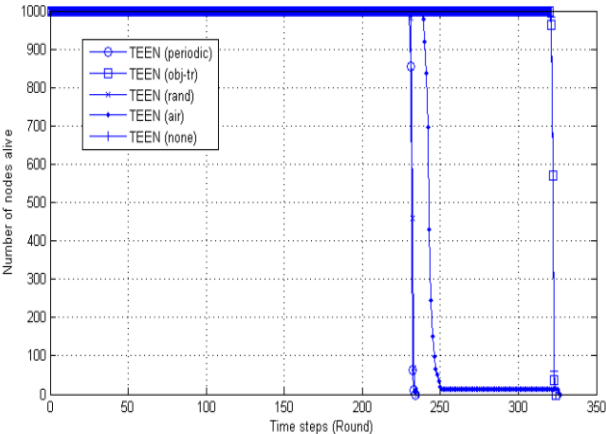

(b)

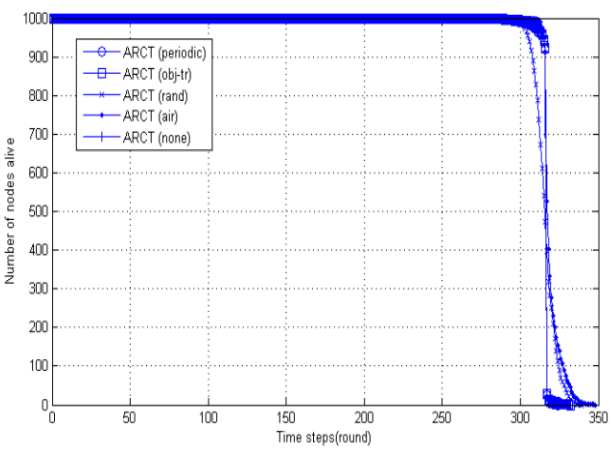

(d)

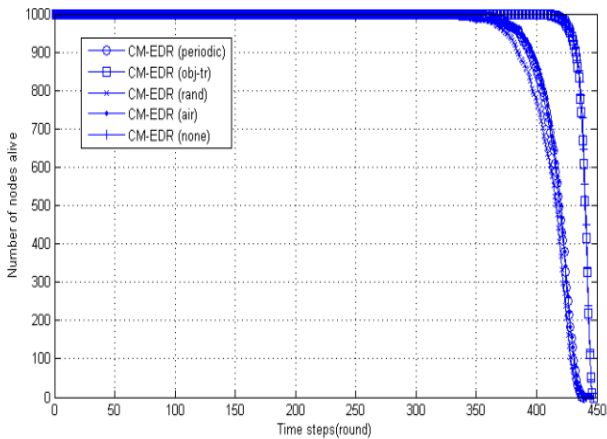

(f)

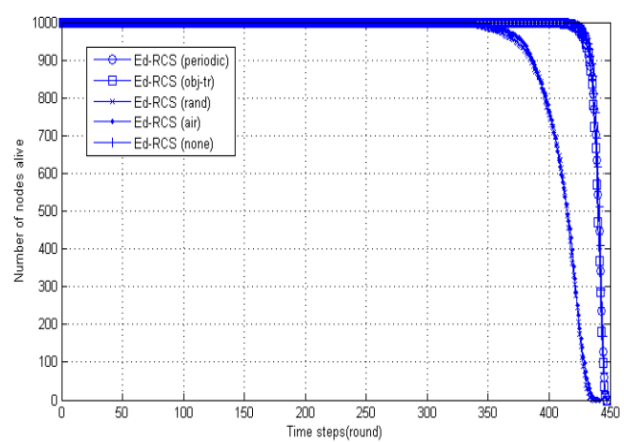

(g)

Fig. 12. Network lifetime comparison. (a) LEACH, (b) TEEN, (c) APTEEN, (d) ARCT, (e) ARCS, (f) $\mathrm{CM}-\mathrm{EDR}$, and (g) Ed-RCS. 


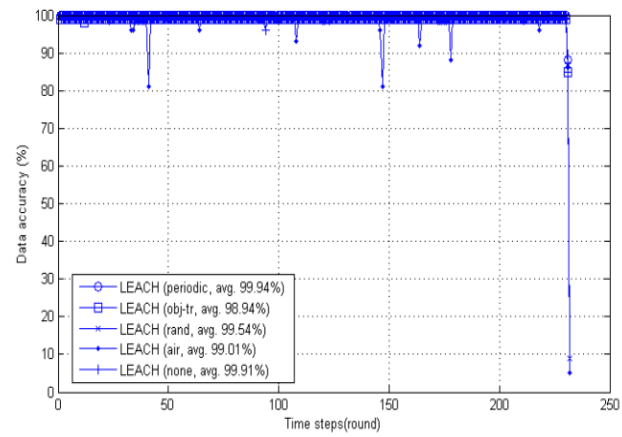

(a)

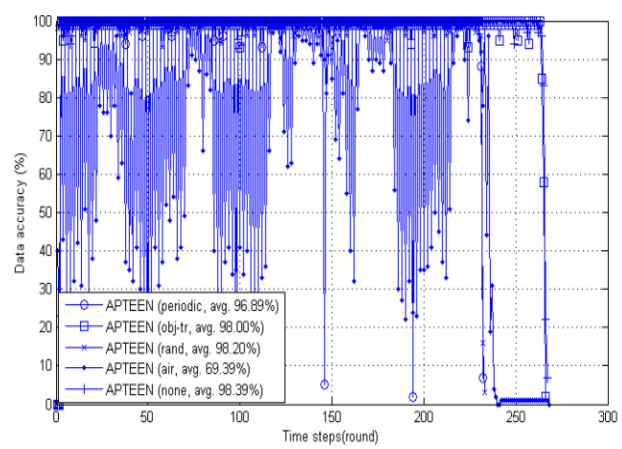

(c)

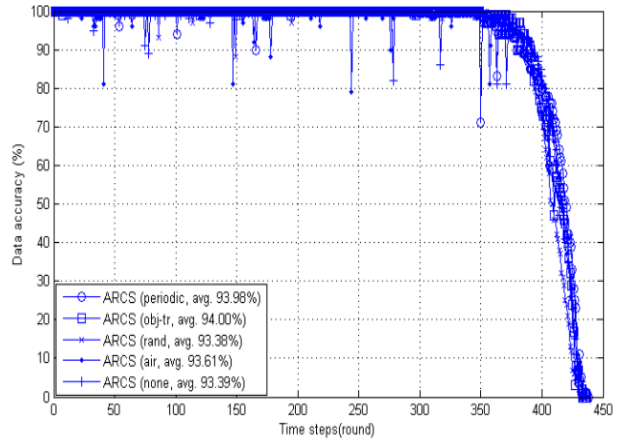

(e)

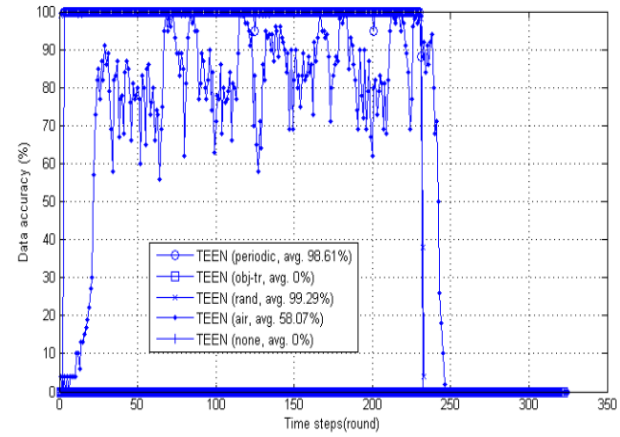

(b)

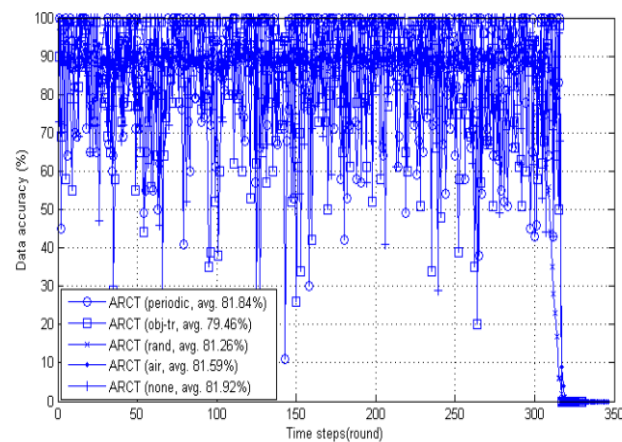

(d)

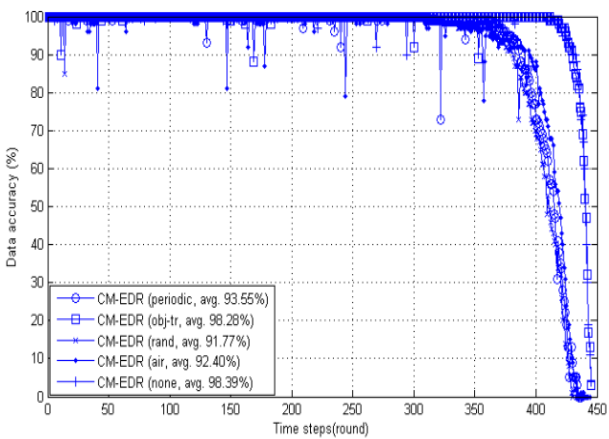

(f)

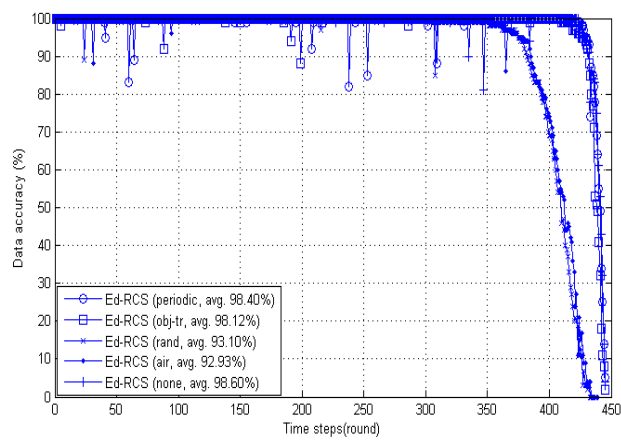

(g)

Fig. 13. Data accuracy comparison. (a) LEACH, (b) TEEN, (c) APTEEN, (d) ARCT, (e) ARCS, (f) CM-EDR, and (g) Ed-RCS. 


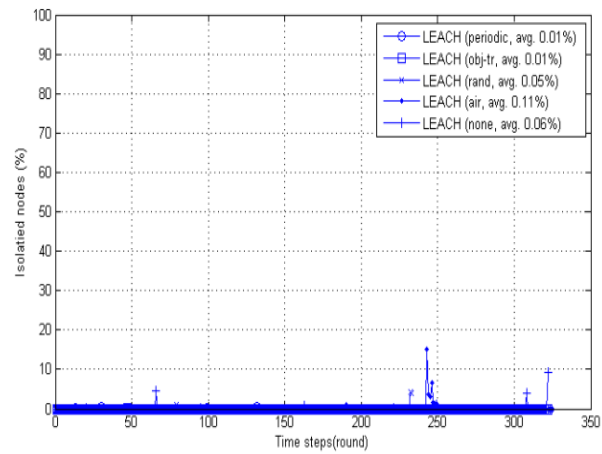

(a)

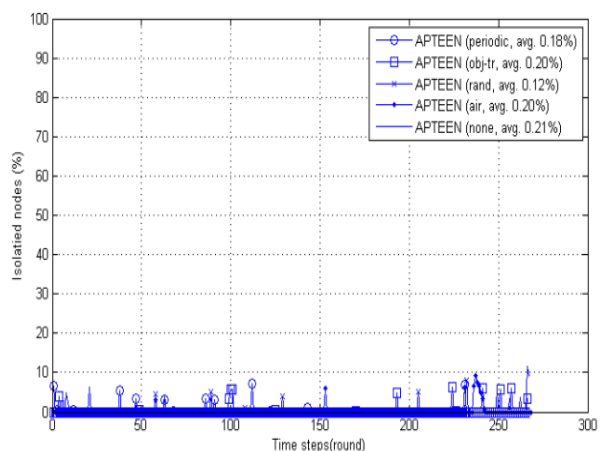

(c)

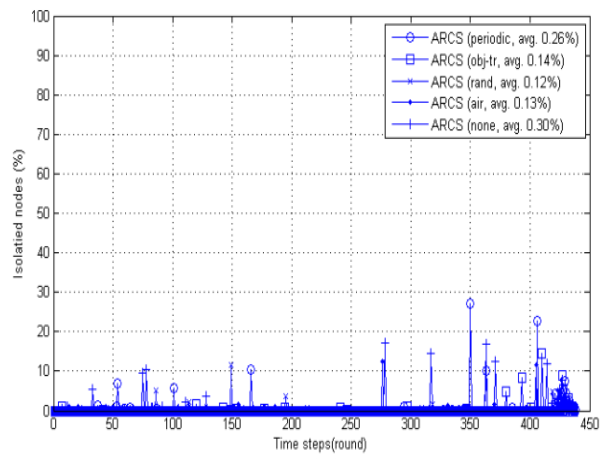

(e)

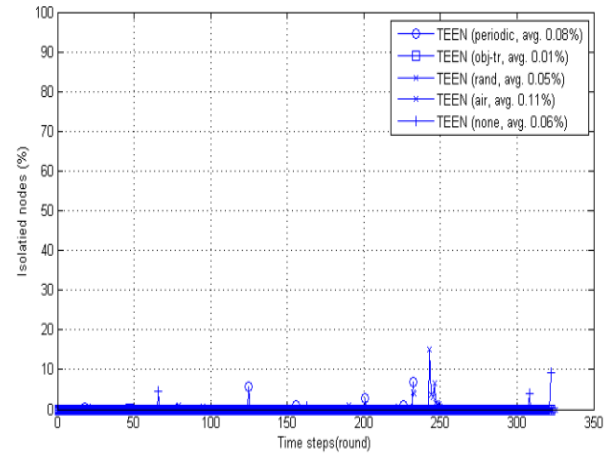

(b)

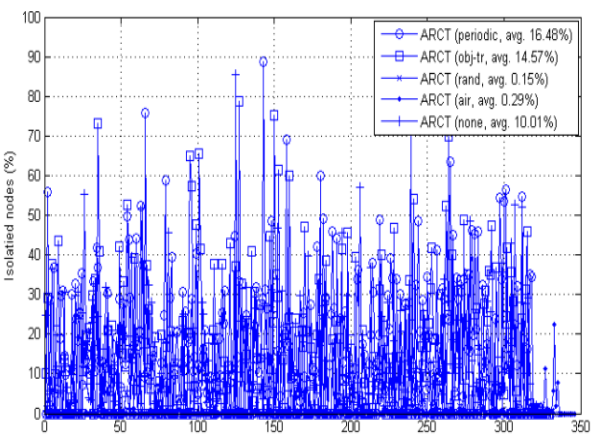

(dime stepss(roun

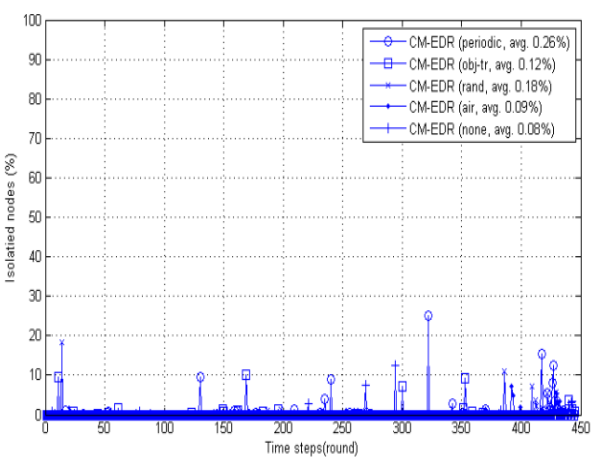

(f)

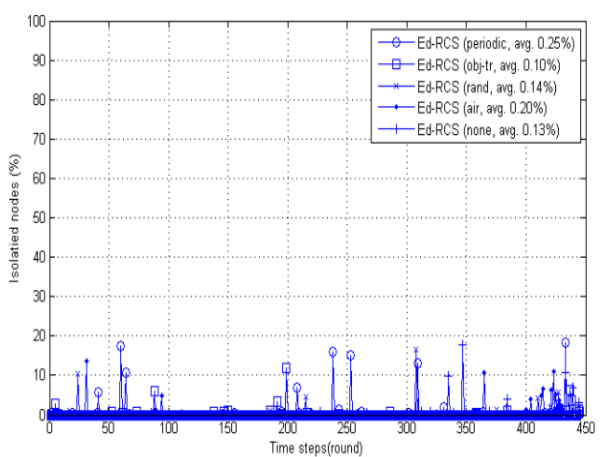

$(\mathrm{g})$

Fig. 14. Node isolation comparison. (a) LEACH, (b) TEEN, (c) APTEEN, (d) ARCT, (e) ARCS, (f) CM-EDR, and (g) Ed-RCS. 


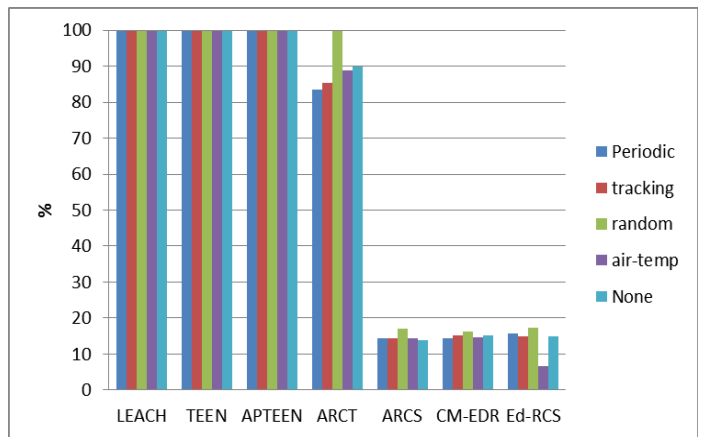

Fig. 15. Node participation probability comparison.

\subsection{Node participation comparison}

Figure 15 shows network participation probability of nodes in each clustering scheme.

LEACH shows high node participation. That means highest data accuracy. On the contrary, in spite of the high node participation of TEEN and APTEEN, they have low data accuracy. Main reason is threshold values. ARCS, CM-EDR and Ed-RCS show low node participation. Even though they show low probability, it directly not means their data accuracy is low. Because of their advanced clustering algorithm, they show guaranteed operation even in low node participation.

In the case of random data pattern, ARCT, ARCS, CM-EDR, and Ed-RCS show a higher node participation probability. It means that these data processing algorithms may not effectively work to save node energy.

\subsection{Residual energy comparison}

It is the critical issue to conserve the available energy of the node for prolong the network lifetime.

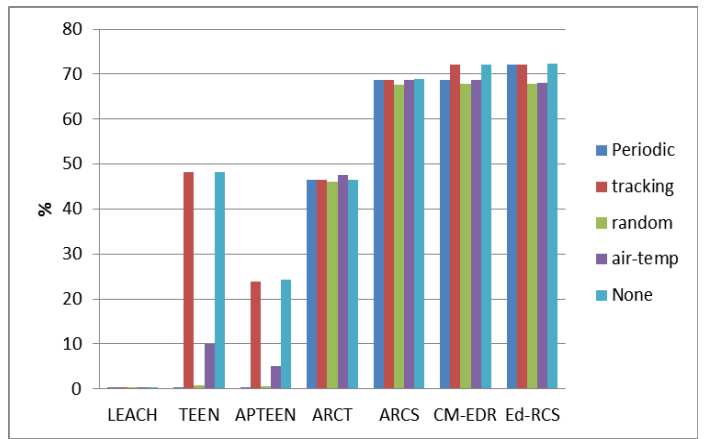

Fig. 16. Residual energy comparison .

Figure 16 shows residual energy comparison that was measured dead node occurred for the first time in LEACH. As shown in figure, each method showed the different results in accordance with data patterns.

In case of LEACH, the similar residual energy is displayed regardless of data patterns. TEEN and APTEEN have higher residual energy than other data patterns in the object tracking and non-data pattern. The other schemes have not been meaningful differences in the residual energy. In the worst case, random data pattern, they show the lowest residual energy.

As mentioned above, in each evaluation comparison category, several schemes were shown to be influenced about data patterns. This shows that previous network performance evaluation is difficult to trust. Table 2 shows how the clustering schemes were influenced by data patterns.

According to table 2, except LEACH, most of the schemes are influenced by data patterns. In particular, network lifetime and data accuracy are significantly influenced by data patterns. Accord-

Table 2. Clustering scheme comparison

\begin{tabular}{|l|c|c|c|c|c|c|c|}
\hline Item & LEACH & TEEN & APTEEN & ARCT & ARCS & CM-EDR & Ed-RCS \\
\hline \hline lifetime & No & Yes & Yes & Little & No & Yes & Yes \\
accuracy & No & Yes & Yes & Yes & Little & Yes & Yes \\
isolation & No & No & No & High & No & No & No \\
participation & No & No & No & High & Little & Little & Little \\
energy & No & Yes & Yes & No & Little & Little & Little \\
\hline
\end{tabular}




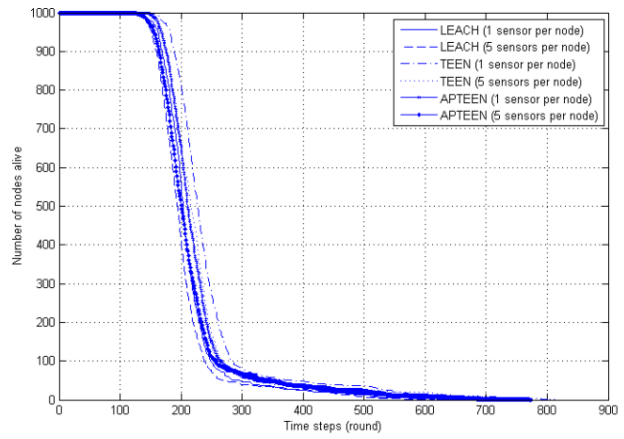

(a)

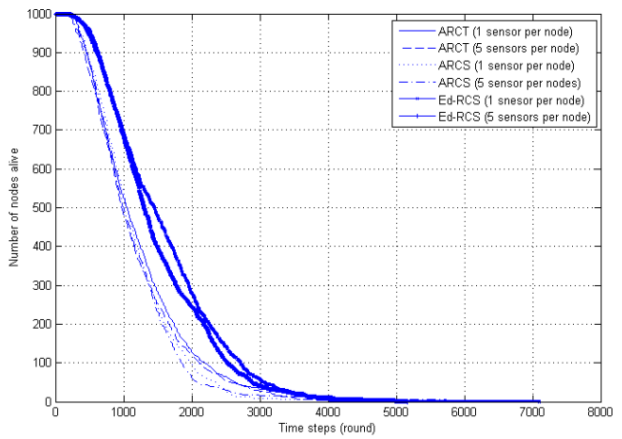

(b)

Fig.17. Sensor network lifetime comparison. (a) LEACH, TEEN, APTEEN, and (b) ARCT, ARCS, Ed-RCS.

ingly, it is strongly suggested that the performance evaluation of clustering schemes should take the data patterns into consideration on the basis of their application areas. Moreover, one or more appropriate data patterns should be used depending on the target applications for fair evaluation and comparison.

\section{8 single vs. multiple sensor data comparison}

Figure 17 shows network lifetime in accordance with the different number of sensors.

As shown in the figure 17, network lifetime is influenced by the number of sensors. However, it seems not serious.

\section{CONCLUSION}

In this paper, we showed it is possible to change the result of the network performance due to sensor and application characteristics. Most of the research papers are premade specified application environment for their sensor network performance evaluation with no more consideration about sensors and environmental characteristics. However, in the case of the universal application environment of the sensor network, various characteristics have to be considered. As shown in the result of the network performance evaluation, sensor characteristic and application environment significantly affect the sensor network widely, and this cannot be ignored.

\section{REFERENCE}

[1] Joseph M. Kahn, Randy H. Katz, and Kristofer S.J. Pister, "Next Century Challenges: Mobile Networking for Smart Dust," Proceeding of IEEE International Conference on Mobile Computing and Networking, pp. 271-278, 1999.

[2] I.F Akyildiz, S. Weilian, Y. Sankarasubramaniam, and E. Cayirci, "A Survey on Sensor Networks," IEEE Communications Magazine, Vol. 40, Issue 8, pp. 102-114, 2002.

[3] Yang-Ick Joo and Jae-Wan Kim, "A Study on Environment Management System in Tunnel using Wireless Sensor networks," Journal of Korea Multimedia Society, Vol. 16, No. 10, pp. 1196-1203, 2013.

[4] G.J. Pottie and W.J. Kaiser, "Wireless integrated Network Sensors," Communications of the ACM, Vol. 43, No. 5, pp. 51-58, 2000.

[5] Y. Yao and J. Gehrke, "Query Processing for Sensor Networks," Proceeding of Conference Innovative Data Systems Research, pp. 1-12, 2003.

[6] Y.C. Tseng, S.Y. Ni, Y.S. Chen, and J.P. Sgeu, "The Broadcast Storm Problem in a Mobile ad Hoc Network," The Journal of Mobile Communication Computation and Information, Vol. 8, No. 2, pp. 153-167, 2002.

[7] J. Li and P. Mohapatra, "An Analytical Model 
for the Energy Hole Problem in Many-to-one Sensor Networks," Proceeding of Vehicular Technology Conference IEEE 62nd, Vol. 4, pp. 2721-2725, 2005.

[8] S.H. Hong and B.K. Kim, "An Efficient Data Gathering Routing Protocol in Sensor Networks using the Integrated Gateway Node," IEEE Transactions on Consumer Electronics, Vol. 56, Issue 2, pp. 627-632, 2010.

[9] W.B. Heinzelman, A.P. Chandrakasan, and H. Balakrishnan, "An Application-specific Protocol Architecture for Wireless Microsensor Networks," IEEE Transactions on Wireless Communications, Vol. 1, No. 4, pp. 660-670, 2002.

[10] O. Younis and S. Fahmy, "Distributed Clustering in Ad-hoc Sensor Networks: a Hybrid, Energy-efficient Approach," Proceedings of IEEE INFOCOM, pp. 629-640, 2004.

[11] S.D. Muruganathan, D. Ma, R. Bhasin and A. Fapojuwo, "A Centralized Energy-efficient Routing Protocol for Wireless Sensor Networks," IEEE Communications Magazine, Vol. 43, No. 3, pp. s8-s13, 2005.

[12] A. Manjeshwar and D.P. Agrawal, "TEEN: a Routing Protocol for Enhanced Efficiency in Wireless Sensor Networks," Proceedings of International Parallel and Distributed Processing Symposium., pp, 2009-2015, 2001.

[13] A. Manjeshwar and D.P. Agarwal, "APTEEN: a Hybrid Protocol for Efficient Routing and Comprehensive Information Retrieval in Wireless Sensor Networks," Proceeding of Parallel and Distributed Processing Symposium, Proceedings International, pp. 195202, 2002.

[14] D. Choi, S. Moh, and I. Chung, "Regional
Clustering Scheme in Densely Deployed Wireless Sensor Networks for Weather Monitoring Systems," The 12th IEEE International Conference on High Performance Computing and Communications, pp. 497-502, 2010.

[15] D. Choi, S. Moh, and I. Chung, "ARCS: An Energy-Efficient Clustering Scheme for Sensor Network Monitoring Systems," ISRN Communications and Networking, Vol. 2011, Article ID 572572, 2011.

[16] T. Kaur and J. Baek, "A Strategic Deployment and Cluster-Header Selection for Wireless Sensor Networks," IEEE Transactions on Consumer Electronics, Vol. 55, No. 4, pp. 1890-1897, 2009.

[17] N. Bouabdallah, M. Rivero-Angeles and B. Sericola, "Continuous Monitoring using Event-Driven Reporting for Cluster- Based Wireless Sensor Networks," Vehicular Technology, IEEE Transactions on, Vol. 58, No. 7, pp. 3460-3479, 2009.

[18] D. Choi, Y. Cho, D. Tak, J. Kang and I. Chung, "A Study of Event-driven Regional Clustering Scheme for Wireless Sensor Networks," Proceeding of The Spring Conference of Korea Multimedia Society, Vol. 14, No. 1, p. 65, 2011.

[19] D. Choi, J. Shen and I. Chung, "A Study of Performance Variation by Sensor Coverage in Wireless Sensor Networks," Proceeding of The Fall Conference of the Korea Multimedia Society, Vol. 14, No. 2, p. 38, 2011.

[20] D. Choi, S. Moh, and I. Chung, "Impact of Sensor Data Patterns on Performance Evaluation of Clustering Schemes in Wireless Sensor Networks," Sensor Letters, Vol. 11, No. 2, pp. 249-258, 2013. 


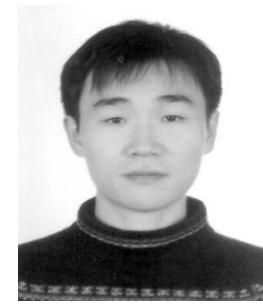

\section{Dongmin Choi}

He received his B.E. degree from the Kyunghee University in 2003 and M.S. and Ph.D. degrees in computer Science from Chosun University in 2007 and 2011, respectively. Since 2014, he has been a Professor in College of General Education, Gwangju, Korea. His research interests are in information security, sensor network systems, mobile ad-hoc systems, smart grid home network systems and internet ethics.

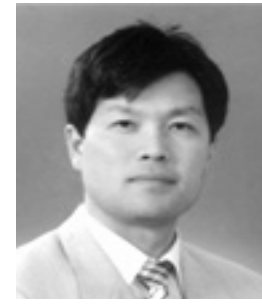

\section{Ilyong Chung}

He received the B.E. degree from Hanyang University, Seoul, Korea, in 1983 and the M.S. and Ph.D. degrees in Computer Science from City University of New York, in 1987 and 1991, respectively. From 1991 to 1994, he was a senior technical staff of Electronic and Telecommunication Research Institute (ETRI), Dajeon, Korea. Since 1994, he has been a Professor in Department of Computer Science, Gwangju, Korea. His research interests are in computer networking, security systems and coding theory. 